\title{
Strain-dependent electrical resistance of multi-walled carbon nanotube/polymer composite films
}

\author{
Myounggu Park ${ }^{1,4}$, Hyonny Kim ${ }^{2,5}$ and Jeffrey P Youngblood ${ }^{3}$ \\ ${ }^{1}$ School of Aeronautics and Astronautics and Birck Nanotechnology Center, \\ Purdue University, IN 47906, USA \\ ${ }^{2}$ Department of Structural Engineering, University of California, San Diego, CA 92093, USA \\ ${ }^{3}$ School of Materials Engineering, Purdue University, IN 47906, USA \\ E-mail: hyonny@ucsd.edu
}

Received 9 August 2007, in final form 28 November 2007

Published 14 January 2008

Online at stacks.iop.org/Nano/19/055705

\begin{abstract}
The strain-dependent electrical resistance characteristics of multi-walled carbon nanotube (MWCNT)/polymer composite films were investigated. In this research, polyethylene oxide (PEO) is used as the polymer matrix. Two representative volume fractions of MWCNT/PEO composite films were selected: $0.56 \mathrm{vol} \%$ (near the percolation threshold) and $1.44 \mathrm{vol} \%$ (away from the percolation threshold) of MWCNT. An experimental setup which can measure electrical resistance and strain simultaneously and continuously has been developed. Unique and repeatable relationships in resistance versus strain were obtained for multiple specimens with different volume fractions of MWCNT. The overall pattern of electrical resistance change versus strain for the specimens tested consists of linear and nonlinear regions. A resistance change model to describe the combination of linear and nonlinear modes of electrical resistance change as a function of strain is suggested. The unique characteristics in electrical resistance change for different volume fractions imply that MWCNT/PEO composite films can be used as tunable strain sensors and for application into embedded sensor systems in structures.
\end{abstract}

(Some figures in this article are in colour only in the electronic version)

\section{Introduction}

Future transportation vehicles are likely to adopt sensor/actuator embedded composite structures to enhance performance and to monitor the health of the structure with the intention of reducing maintenance costs [1]. Such a composite structure is usually called a 'smart' structure, due to its capability of sensing and responding to the surrounding environmental situation. Among the many functions being pursued to incorporate into the host composite structure, strain sensing is one of the most basic ones. The main reason for developing an embedded strain sensing system in a composite structure using thin and compact films is that it allows one to measure the

\footnotetext{
4 Department of Mechanical Engineering, University of Michigan, Ann Arbor, MI 48109, USA (new affiliation after September/1/2007).

5 Author to whom any correspondence should be addressed.
}

static and dynamic response [2, 3] without significant adverse effects on the host structure.

Recently, a large body of research about carbon nanotube (CNT) embedded polymer composites has been conducted [4] due to CNTs possessing excellent stiffness and strength properties that are desirable attributes as a reinforcing fiber. In addition to those outstanding mechanical properties, many superior and unique characteristics including electrical conductivity, thermal conductivity, Raman active properties, and electrical property change induced by strain make CNT embedded polymer composites ideal for application into smart structures [5-10]. For example, Frogley et al [10] embedded CNTs into a matrix and detected the Raman spectrum shift as a function of strain. Dharap et al [11] demonstrated that a CNT filled polymer film can be used as a strain sensor by measuring the electrical resistance induced by strain. Those reported studies using CNT composite films as a strain sensor 


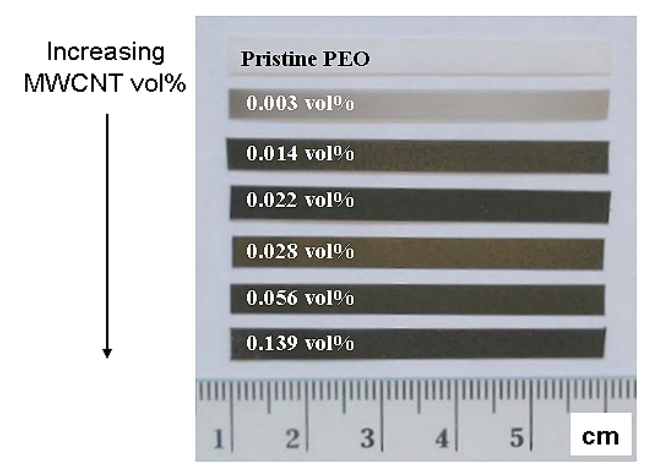

(a) Fabricated MWCNT/PEO Composite Films

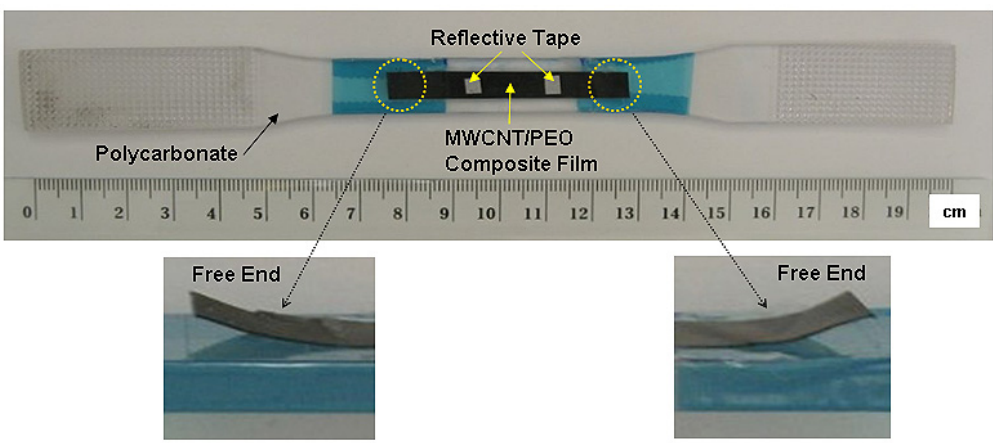

(b) Film Bonded to Polycarbonate Substrate

Figure 1. MWCNT/PEO film and tensile dogbone specimen.

showed quite successful and promising results. However, at present, overall research on CNT embedded polymer as a strain sensor is in the early stages. The topic of this paper is focused on the measurement, physical understanding and modeling of electrical resistance change in CNT/polymer composite films. In this research, the matrix is PEO but the results are generally applicable to MWCNT embedded in polymer. The MWCNT/PEO composite films used in the present study are fabricated by intentional coagulation of dispersed MWCNTs and PEO $[12,13]$. Also, the application of MWCNT/polymer composite films as a strain sensing device is discussed.

\section{Experiments}

\subsection{Experimental setup}

To investigate the relationship between electrical resistance and strain for MWCNT/PEO composite films, two representative volume fractions were tested: $0.56 \mathrm{vol} \%$ (near the percolation threshold) and 1.44 vol\% (away from the percolation threshold) of MWCNT. Examples of fabricated films are shown in figure 1(a). The fabrication procedure and the experimental determination of the percolation threshold of these films are described in $[12,13]$. The percolation threshold is observed to be between 0.14 and 0.28 vol\% of MWCNT (0.5 and $1 \mathrm{wt} \%$ of MWCNT). From each film fabricated at select volume fractions, two strip specimens were taken.

Dogbone shaped polycarbonate plastic tensile test specimens were prepared and the MWCNT/PEO composite

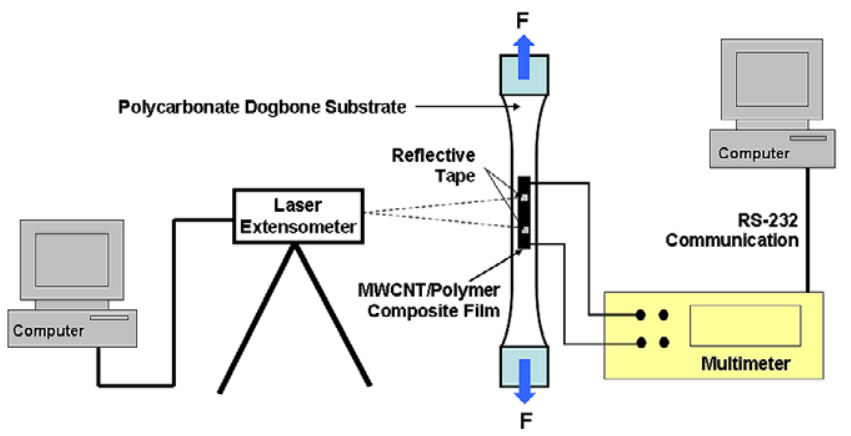

Figure 2. Electrical resistance and strain measurement.

film strips of 0.56 and 1.44 vol\% MWCNT were bonded onto the center of the gage section using strain gage adhesive (M Bond 200) as shown in figure 1(b). The polycarbonate dogbone substrate has gage section dimensions of $50 \mathrm{~mm}$ by $13 \mathrm{~mm}$, and thickness $2.92 \mathrm{~mm}$. The overall test setup is shown in figure 2. The combined film bonded polycarbonate dogbone was stretched using a uniaxial test machine (MTS 810). The strain of the combined specimen was recorded using a laser extensometer (Electronics Instrument Research, Ltd; Model LE-05) while the resistance of the MWCNT/PEO film was simultaneously measured using a precision multimeter (Keithley 2000). Since the MWCNT/PEO film is well bonded to the substrate and has much lower stiffness, the strain in the film is the same as the strain in the polycarbonate substrate. 


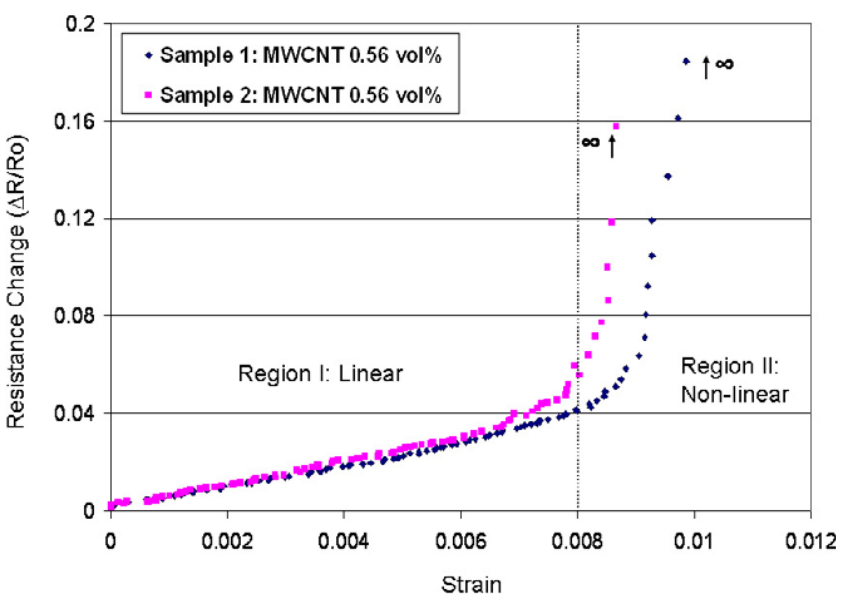

Figure 3. Resistance versus strain for 0.56 vol\% of MWCNT.

To make sure that the resistance change was induced only by the deformation of the composite film and not changes in wire contact quality, the end regions of the film were not bonded to the dogbone specimen and the lead wires were then attached to these unstretched end regions (see figure 1(b)). To make a secure contact of the lead wires to the cross-section of the film, the lead wires were heated to $50^{\circ} \mathrm{C}$ and then gently pressed onto the film surface, thereby embedding them into the crosssection. Conductive silver paste adhesive (Loctite 3888) was then applied over this connection. Although the two-point measurement scheme includes the resistance of lead wires and contacts, this added resistance is negligible relative to the resistance range of the films which is greater than $1 \Omega$ [14].

\subsection{Strain-dependent electrical resistance}

The initial electrical resistances (i.e., with no externally applied strain) were 598 and $493 \mathrm{k} \Omega$ for the two specimens having $0.56 \mathrm{vol} \%$ of MWCNT, and 6.89 and $6.29 \mathrm{k} \Omega$ for the specimens having 1.44 vol\% of MWCNT. To compare the relationship between electrical resistance versus strain, the change in electrical resistance $\Delta R / R_{0}$ is plotted against strain in figures 3 and 4 , where $\Delta R$ is the difference between the current resistance $(R)$ and initial resistance $\left(R_{0}\right)$. For the $0.56 \mathrm{vol} \%$ MWCNT films (see figure 3), the electrical resistance increased in a linear and monotonic manner up to 0.008 strain, and then transitioned to a nonlinear behavior, rapidly increasing in electrical resistance just before 0.009 strain. For the 1.44 vol\% MWCNT films (see figure 4), the electrical resistance increased linearly over a larger strain range (0.01-0.02) compared to the 0.56 vol\% MWCNT case. After 0.02 strain, the relationship between electrical resistance and strain became nonlinear. The measurements for the $1.44 \mathrm{vol} \%$ MWCNT films were stopped due to the onset of the localized necking of the polycarbonate substrate at about 0.07 strain (see figure 5).

The relationship between electrical resistance and strain can be divided into a linear and nonlinear region for both the 0.56 and 1.44 vol\% MWCNT cases, as indicated in figures 3 and 4 . The linear and nonlinear region was more distinctive for

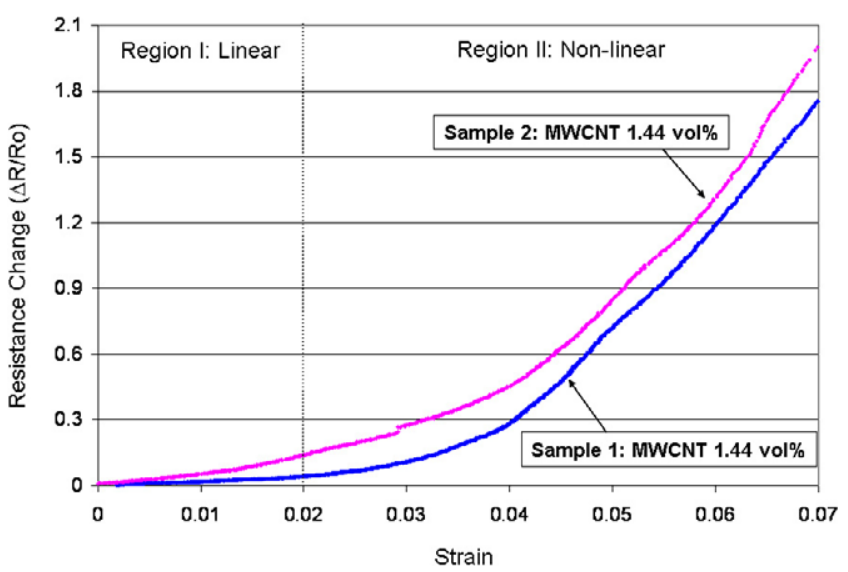

Figure 4. Resistance versus strain for 1.44 vol\% of MWCNT.

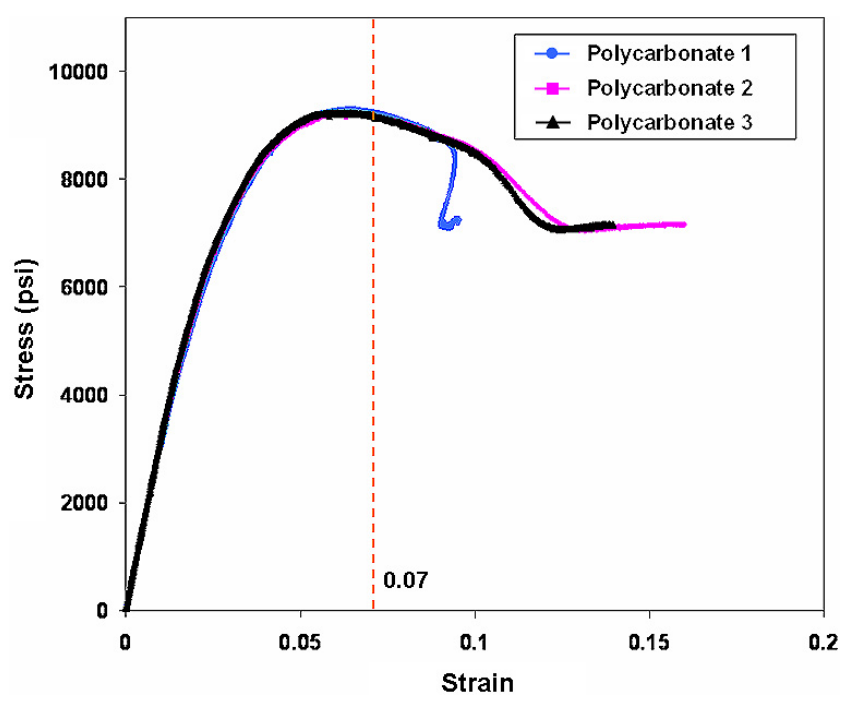

Figure 5. Tensile test results of polycarbonate substrate.

0.56 vol\% MWCNT specimens compared with the 1.44 vol\% MWCNT specimens. However, the overall pattern of electrical resistance change versus strain for the specimens of each volume fraction of MWCNT was found to be quite repeatable. The reasons for the linear to nonlinear transition in electrical resistance change as a function of strain are discussed in the following sections.

\section{Discussion}

\subsection{Electrical resistance versus microstructure change}

As a MWCNT/PEO composite specimen is stretched, the initial microstructure of the material changes, thereby affecting the number of MWCNT-to-MWCNT contacts. This is a result of the initially random network of MWCNTs becoming more ordered. The microstructure change of a 2.99 vol\% MWCNT specimen was directly observed before and after straining by a field-emission scanning electron microscope (FE-SEM). The amount of strain applied to the specimen 


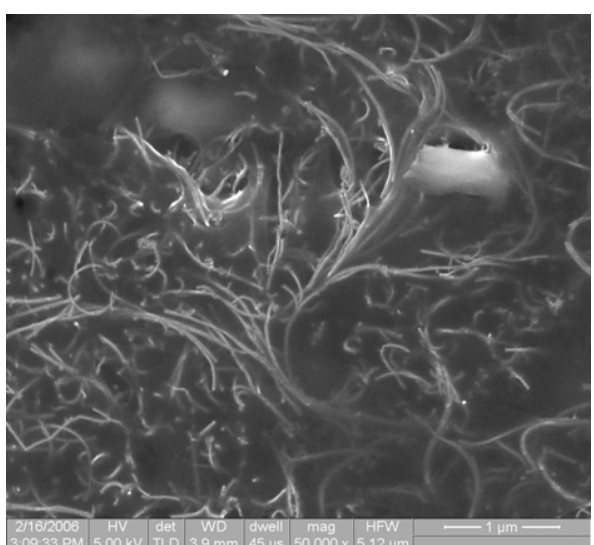

Figure 6. Microstructure of MWCNT/PEO composite before straining.

was approximately $100 \%$, which is much larger than the levels shown in figures 3 and 4, in order to obtain better observation. As shown by comparing the unstretched film (see figure 6) with the stretched film (see figure 7), the initially non-directionally embedded MWCNTs become straight and aligned with the direction of stretching. It is now argued that the number of MWCNT-to-MWCNT contacts reduces with increasing directional alignment of the nanotubes, thereby causing an increase in the electrical resistance. Furthermore, it is hypothesized that for a specimen filled with MWCNTs well above the percolation threshold, the reduction in number of MWCNT-to-MWCNT contacts is less severe than for a specimen having MWCNT content near the percolation threshold, when both are stretched under the same amount of applied strain. Thus, by varying the MWCNT volume fraction, unique strain-dependent electrical resistance change characteristics can be obtained.

\subsection{Electrical resistance change model}

MWCNTs inside the matrix can be considered as overlapping at the contact locations, rather than being arranged in an endto-end configuration. This affects how the tunneling distance changes, as depicted simply in figure 8 . For higher volume

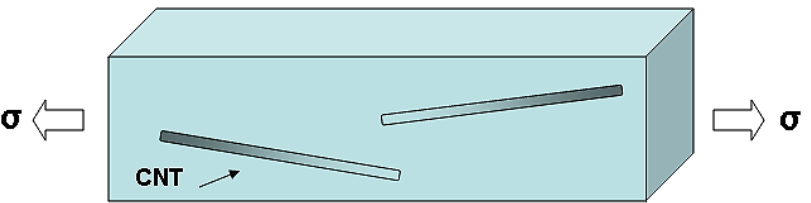

(a) Lower Volume Fraction Specimen

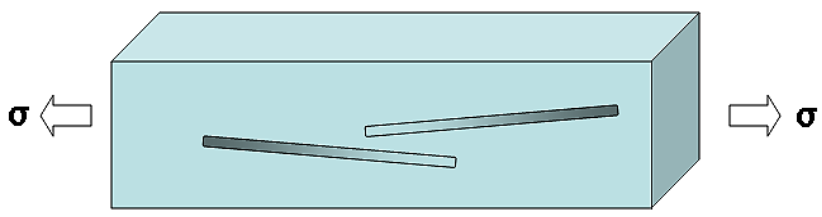

(b) Higher Volume Fraction Specimen

Figure 8. Contact geometry of MWCNTs inside a matrix.

fraction MWCNT/PEO films, there will generally be more overlap, and so the tunneling resistance would not play a role until larger levels of strain. Based on this description, a resistance change model is proposed by superposing a modified percolation-based scaling rule [15-17] (related to the linear resistance change region) with relationships describing tunneling resistance (related to the nonlinear resistance change region). This model is expressed as

$$
\frac{\Delta R}{R_{0}}=\frac{\Delta R_{\mathrm{L}}+\Delta R_{\mathrm{N}}}{R_{0}}=\frac{\Delta R_{\mathrm{L}}}{R_{0}}+\frac{\Delta R_{\mathrm{N}}}{R_{0}}
$$

where $\Delta R_{\mathrm{L}}$ is resistance change in the linear region, $\Delta R_{\mathrm{N}}$ is the resistance change in the nonlinear region, and $R_{0}$ is the initial bulk electrical resistance. In the linear region, the MWCNTs are still overlapping (i.e., in contact) and the scaling rule is applicable [15-17]. A percolation-based scaling rule [13] predicting bulk electrical conductivity, $G$, has been modified to account for the effects of volume change of a specimen on the MWCNT volume fraction $\Phi$ and percolation threshold volume fraction $\Phi_{c}$. This is expressed as

$$
G=K\left[\frac{1}{c} \Phi-c \Phi_{\mathrm{c}}\right]^{t}
$$

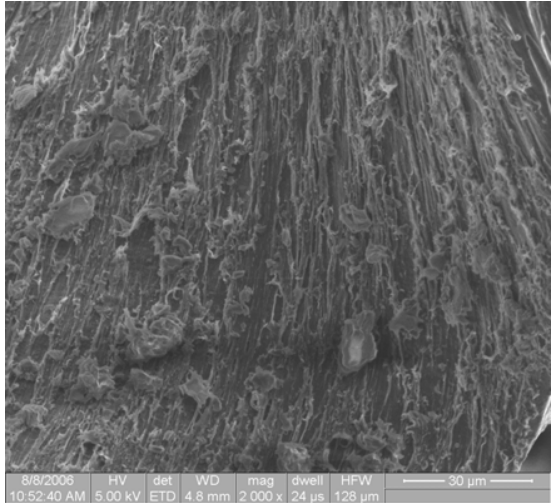

(a) Large Area View

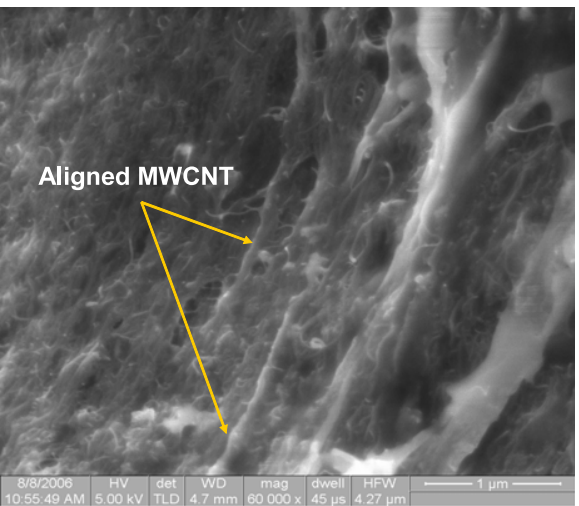

(b) Close View

Figure 7. Microstructure of 5.9 vol\% MWCNT/PEO composite after straining. 


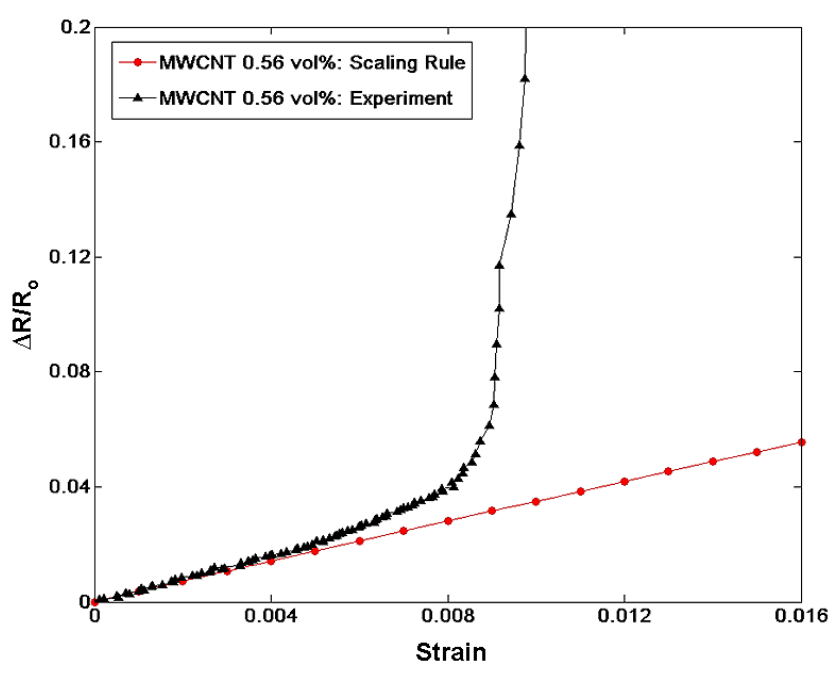

(a) MWCNT 0.56 vol $\%$

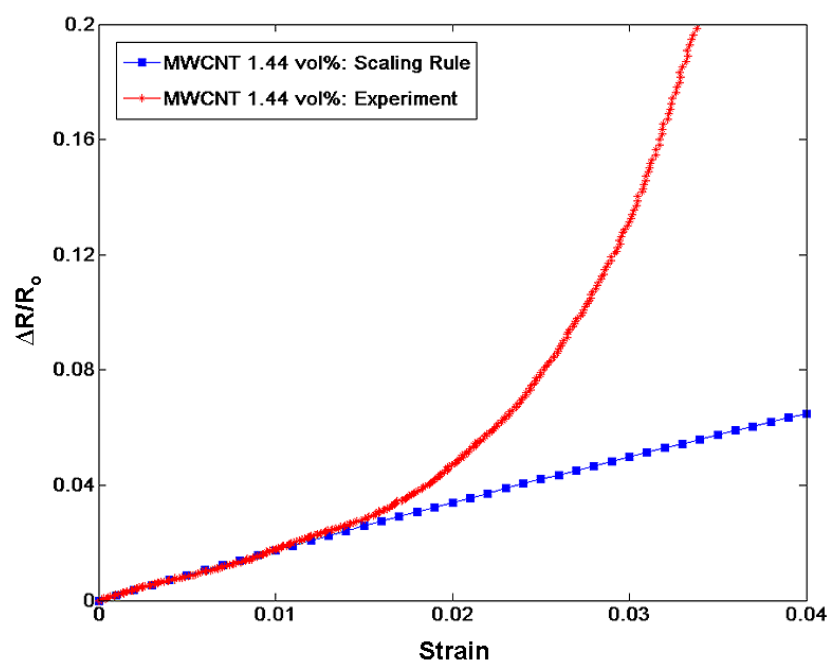

(b) MWCNT 1.44 vol\%

Figure 9. Electrical resistance change versus strain.

where $K$ is a constant, $t$ is the critical conductivity exponent, measured to be 2.47 [13], and $c$ is equal to $V / V_{0}$, the ratio of present volume to initial volume when a material is subjected to a uniaxial strain $\varepsilon_{x}$ (equation (3)).

$$
\frac{V}{V_{0}}=\left(1+\varepsilon_{x}\right)\left(1-v_{x y} \varepsilon_{x}\right)\left(1-v_{x z} \varepsilon_{x}\right)
$$

$\Delta R / R_{0}$ versus strain predicted by equation (2) is found to be in good agreement with the experimental data over the linear region, as shown in figure 9 , when $\Phi_{\mathrm{c}}$ is chosen as $0.28 \mathrm{vol} \%$.

For strain levels beyond the linear region, equation (2) cannot predict the experimental data. In this nonlinear region, MWCNTs are hypothesized to be losing overlapping contact with each other, and thus tunneling resistance [18-20] becomes a dominant phenomenon. The transition from the linear to nonlinear region, i.e., the strain at which the numbers of contacts start reducing significantly, is defined as critical

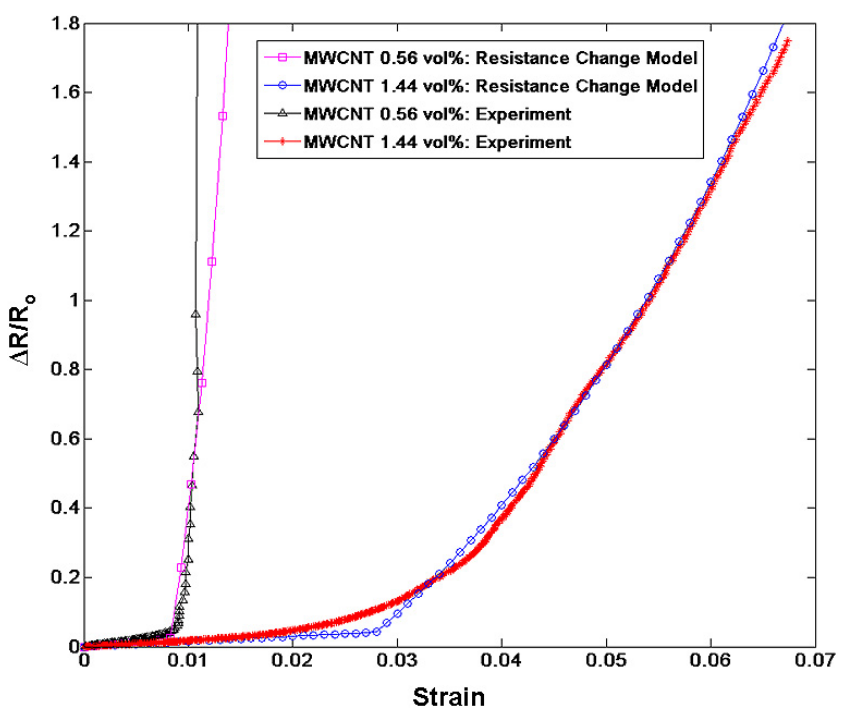

Figure 10. Resistance change model versus experiments.

strain $\varepsilon^{c}$. Tunneling resistance changes [20] are expressed as

$$
\begin{aligned}
\frac{\Delta R_{\mathrm{N}}}{R_{0}} & \approx \exp \left(\frac { 4 \pi \sqrt { 2 m \beta } } { h } \langle s _ { 0 } \rangle ( \varepsilon - \varepsilon ^ { \mathrm { c } } ) \left(\cos ^{2}\langle\eta\rangle\right.\right. \\
& \left.\left.-v \sin ^{2}\langle\eta\rangle\right)\right)-1
\end{aligned}
$$

where $h$ is Plank's constant $\left(6.62 \times 10^{-34} \mathrm{~J}\right.$ s $)[21], \beta$ is the work function of a CNT $(4.8 \mathrm{eV})[22,23], m$ is the mass of an electron $\left(9.11 \times 10^{-31} \mathrm{~kg}\right)[21],\left\langle s_{0}\right\rangle$ is the average distance between MWCNTs, and $\langle\eta\rangle$ is the average angle between the direction of applied strain and the tunneling path, calculated as $\langle\eta\rangle=\sin ^{-1} \frac{\pi}{4}$ [24]. By superposing the resistance change of the linear and nonlinear regions, the following equation is proposed for predicting the full range of strain-dependent resistance of the MWCNT/PEO composite film.

$$
\frac{\Delta R}{R_{0}} \approx \begin{cases}k \varepsilon & \left(0 \leqslant \varepsilon \leqslant \varepsilon^{\mathrm{c}}\right) \\ k \varepsilon+u\left(\varepsilon-\varepsilon^{\mathrm{c}}\right) N & \left(\varepsilon>\varepsilon^{\mathrm{c}}\right)\end{cases}
$$

where $k$ is the slope of linear region determined using a modified scaling rule and $N$ is the right-hand side of equation (4). The best fit to the data by equation (5) is obtained when $\left\langle s_{0}\right\rangle$ and $\varepsilon^{\mathrm{c}}$ are $600 \AA$ and 0.0083 for the $0.56 \mathrm{vol} \%$ film, and $85 \AA$ and 0.028 for the 1.44 vol\% film. Also $k$ is calculated to be 3.12 and 1.5 for the 0.56 and $1.44 \mathrm{vol} \%$ films, respectively. Predictions using equation (5) compared with experimental data are shown in figure 10.

Equation (5) is capable of describing strain-dependent resistance reasonably well except at the transition region near the critical strain. The resistance change model suggested here for MWCNT/PEO composite film is a highly simplified model and assumes that the MWCNTs are straight, whereas they are actually wavy. Thus, the effect of MWCNT shape on the percolation threshold $\left(\Phi_{\mathrm{c}}\right)$ should be considered. Additionally, anomalies like voids, cracks, and non-uniformity of the film thickness over the volume of the specimen tested should be accounted for since they affect stress distributions and ultimately the overall deformation-dependent electrical resistance behavior. 
Table 1. Strain sensitivity comparison

\begin{tabular}{|c|c|c|c|c|}
\hline \multirow{3}{*}{$\begin{array}{l}\text { Metal alloys } \\
\text { used for foil } \\
\text { type strain gage }\end{array}$} & \multicolumn{4}{|c|}{ MWCNT/PEO composite film } \\
\hline & \multicolumn{4}{|c|}{ MWCNT 0.56 vol\% MWCNT 1.44 vol\% } \\
\hline & Region I & Region II & Region I & Region II \\
\hline $0.74-5.1$ & 3.7 & - & 1.6 & 50 \\
\hline
\end{tabular}

\subsection{Application as strain sensor}

The MWCNT/PEO composite film reported herein showed repeatable and tunable electrical resistance versus strain relationships for different volume fractions of MWCNT. Application of this material as a strain sensor is now considered. Strain sensitivity is one of the key performance descriptors for strain sensing materials, and is defined [25] as

$$
S=\frac{\Delta R / R_{0}}{\varepsilon} .
$$

The strain sensitivity for the two tested MWCNT/PEO specimens and the strain sensitivity of metal alloys [26] typically used for conventional foil type strain gages are shown in table 1. In the linear region (region I), the strain sensitivity of the specimens is comparable to that of metal alloys used for conventional foil gages. In the nonlinear region (region II), the 1.44 vol\% specimen showed much higher strain sensitivity than the conventional foil gage. The 1.44 vol\% MWCNT/PEO film has been shown to be operational over a wide strain range, beyond 0.07 (limited by polycarbonate dogbone substrate necking failure), whereas previous works $[10,27,28]$ have reported measurements up to a strain of 0.04 . For the $0.56 \mathrm{vol} \%$ case, the abrupt change in resistance suggests that it can function like a switch. A useful linear strain range for the $0.56 \mathrm{vol} \%$ film is up to 0.008 strain and a useful strain range for the $1.44 \mathrm{vol} \%$ film is beyond 0.07 . Note that this can be even higher since the test was stopped due to the onset of the localized necking of the polycarbonate dogbone substrate after 0.07 strain.

\section{Conclusions}

In the current research, MWCNT/polymer composite films are used to investigate macroscale deformation-dependent electrical resistance change. The following conclusions are made.

(1) The deformation-dependent electrical resistance of MWCNT/PEO is unique and repeatable for different volume fractions of MWCNT. The overall pattern of electrical resistance change versus strain for the specimens of each volume fraction of MWCNT consists of a linear region followed by nonlinear behavior.

(2) In the linear region, the modified scaling rule prediction matched well with the experimental results. It is hypothesized that, in the linear region, the overlapping contact of MWCNTs inside the matrix is maintained during stretching of the film. Thus, the percolation-based theory was found to be applicable. However, after passing the critical strain, the overlapping contact of MWCNTs is hypothesized to be lost, and thus the percolation-based theory breaks down. In the nonlinear region, the tunneling resistance model is found to be suitable for describing the electrical resistance change, thereby supporting the hypothesis that electron tunneling plays a dominant role within the nonlinear region.

(3) The resistance change model combining the modified scaling rule and tunneling resistance was suggested and shown to predict the linear and nonlinear modes of electrical resistance change versus strain reasonably well, except in the transition region.

(4) The unique and repeatable characteristics in electrical resistance change of the MWCNT/PEO films permit these materials to be used as tunable strain sensors and for application into systems such as embedded sensors in composite structures.

\section{Acknowledgment}

This research was supported by the Purdue Research Foundation.

\section{References}

[1] Blanas P and Das-gupta D K 1999 Composite piezoelectric sensors for smart composite structures 10th Int. Symp. on Electrets pp 731-4

[2] Javidinejad A and Joshi S P 1999 Design and structural testing of smart composite structures with embedded conductive thermoplastic film Smart Mater. Struct. 8 585-90

[3] Thostenson E T and Chou T-W 2006 Carbon nanotube networks. Sensing of distributed strain and damage for life prediction and self healing Adv. Mater. 18 2837-41

[4] Qian D, Wagner G J and Liu W K 2002 Mechanics of carbon nanotubes Appl. Mech. Rev. 55 495-533

[5] Dai H, Wong E W and Lieber C M 1996 Probing electrical transport in nanomaterials: conductivity of individual carbon nanotubes Science 272 523-6

[6] Ebbesen T W, Lezec H J, Hiura H, Bennett J W, Ghaemi H F and Thio T 1996 Electrical conductivity of individual carbon nanotubes Nature 382 54-6

[7] Hone J, Whitney M and Zettl A 1999 Thermal conductivity of single-walled carbon nanotubes Synth. Met. 103 2498-9

[8] Fujii M, Zhang X, Xie H, Ago H, Takahashi K, Ikuta T, Abe $\mathrm{H}$ and Shimizu T 2005 Measuring the thermal conductivity of a single carbon nanotube Phys. Rev. Lett. 95065502

[9] Souza Filho A G, Kobayashi N, Jiang J, Grüneis A, Saito R, Cronin S B, Mendes Filho J, Samsonidze Ge G, Dresselhaus G and Dresselhaus M S 2005 Strain-induced interference effects on the resonance Raman cross section of carbon nanotubes Phys. Rev. Lett. 95217403

[10] Frogley M D, Zhao Q and Wagner H D 2002 Polarized resonance Raman spectroscopy of singe-walled carbon nanotubes within a polymer under strain Phys. Rev. B 65113413

[11] Dharap P, Li Z, Nagarajaiah S and Barrera E V 2004 Nanotube film based on single-wall carbon nanotubes for strain sensing Nanotechnology 15 379-82

[12] Park M and Kim H 2006 Evaporation-based method for fabricating conductive MWCNT/PEO composite film and its application as strain sensor 12th US-Japan Conf. on Composite Materials (Dearborn, MI) 
[13] Park M 2007 Deformation dependent electrical resistance of MWCNT layer and MWCNT/PEO composite films $P h D$ Thesis Purdue University, School of Aeronautics and Astronautics

[14] 2004 I-V Measurements of nanoscale wire and tubes with the model 4200-SCS and Zyvex S100 nanomanipulator. Keithley inc. Application Note Series; Number 2418

[15] Stauffer D and Aharony A 1994 Introduction to Percolation Theory 2nd edn (New York: CRC press)

[16] Munson-McGee S H 1991 Estimation of the critical concentration in an anisotropic percolation network Phys. Rev. B 43 3331-6

[17] Balberg I 1986 Excluded-volume explanation of Archie's law Phys. Rev. B 33 3618-20

[18] Simmons J G 1963 Low-voltage current-voltage relationship of tunnel junctions J. Appl. Phys. 34 238-9

[19] Simmons J G 1963 Generalized formula for the electric tunnel effect between similar electrodes separated by a thin insulating film J. Appl. Phys. 34 1793-803
[20] Yasuoka T, Shimamura Y and Todoroki A 2005 Piezorisistivity of carbon-nanotube composite The 9th Japan Int. SAMPE Symp. pp 341-4

[21] Ashcroft N W and Mermin N D 1976 Solid State Physics (Stamford, CT, USA: Thomson Learning)

[22] Masashi S and Masafumi A 2001 Work function of carbon nanotubes Carbon 39 1913-7

[23] Gao R, Pan Z and Wang Z L 2001 Work function at the tips of multiwalled carbon nanotubes Appl. Phys. Lett. 78 1757-9

[24] Onsager L 1949 The effects of shape on the interaction of colloidal particles Ann. New York Acad. Sci. 51 627-59

[25] Hambley A R 2005 Electrical Engineering 3rd edn (New Jersey: Pearson Education)

[26] Efunda engineering fundamentals http://efunda.com/ designstandards/sensors/strain_gages/strain_gage_sensitivity. $\mathrm{cfm}$

[27] Wood J R and Wagner H D 2000 Single-wall carbon nanotubes as molecular pressure sensors Appl. Phys. Lett. 76 2883-5

[28] Li C and Chou T-W 2004 Strain and pressure sensing using single-walled carbon nanotubes Nanotechnology 15 1493-6 